\title{
Waldsterben - Was ist daraus geworden?
}

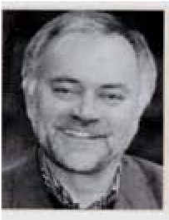

\section{Mario F. Broggi}

Direktor der Eidgenössischen Forschungsanstalt WSL in Birmensdorf und Privatdozent an der Universität für Bodenkultur in Wien

E-Mail: mario.broggi@wsl.ch www.wsl.ch
Abstract: The forest decline predicted in the early eighties fortunately did not occur. Research initiated in these days showed that a quick answer to these extremely complex problems was hardly possible. The forest condition inventories, which are continuing today, emphasize : the importance of long-term research. It is recognized today that air pollution generally causes less acute damage than long-term impact. in der polnisch-tschechoslowakischen Grenze wurde ich 1970 in einem Sattelmoor im Nationalpark Riesengebirge vom Militär angehalten und vernommen. Rübezahls Heimat war damals noch ein geschlossenes Waldmeer, das mich durch seine Ausdehnung beeindruckte. In den späten 1970er und in den 1980er Jahren sind viele dieser Wälder in kurzer Zeit abgestorben [1].

Bilder wie die von toten Wäldern im Dreiländereck Polen-TschechoslowakeiDDR (siehe Abbildung) geisterten anfangs der 1980er Jahre durch die deutsche Presse; ich entsinne mich noch der dramatischen dreiteiligen Titelgeschichte im 'Spiegel' des Jahres 1981. Auch in der Schweiz stellte man, aufgeschreckt durch diese Bilder, Symptome eines Nadel- und Blattverlustes fest, und dies wurde als beginnendes Waldsterben interpretiert. Die Sorge vor allem um unsere Bergwälder war sehr groß, spielt hier doch die Schutzfunktion eine herausragende Rolle. Dies führte $\mathrm{zu}$ Umfragen, ersten Sanasilva-Erhebungen und Interpretationen der Kronenverlichtungen. Der Nationalfonds finanzierte ein großes Forschungsprogramm (NFP 14+). Poli-tische Manifestationen, internationale Konferenzen und Konventionen überstürzten sich. Eine mediale Grundwelle "Waldsterben" schwappte auch über die Schweiz, inklusive deren Wissenschaft. Wissenschaftler und Wissenschaftlerinnen ließen sich teilweise mitreißen. Die Wirkung war so ausgeprägt, daß noch vorhandene $Z$ weifler verstummten.

Das damals prognostizierte Waldsterben ist bei uns zum Glück ausgeblieben, doch die jährlichen europaweiten Walderhebungen, die den Gesundheitszustand der Bäume nach der Dichte der Belaubung beschreiben, sind geblieben. Danach weist bei uns ein Viertel der Bäume deutliche Kronenverlichtung auf. Es ist dies allerdings eine sogenannte "End-of-pipe-Aussage", von der man nicht weiß, was für das Endergebnis der Beobachtung maßgeblich ist, wie es also zustande kommt. Wird das Erscheinungsbild beziehungsweise die Vitalität der Bäume vom Boden, vom Klima und dessen Veränderung, von der Höhenlage, vom Alter der Bäume, von deren Exposition oder von der Luftverschmutzung beeinflußt? Auch der Zusammenhang zwischen Erscheinungsbild und Gesundheit bleibt problematisch und damit forschungswürdig.

Der dem "Waldsterben" folgende Öko-Aufruf zu Verhaltensänderungen bei der Auto-Mobilität fruchtete wenig. Kampagnen wie "Der Wald stirbt, fahr weniger Auto" stempelten die Träger der grünen Bewegung zu Langweilern und Miesmachern. Dabei hätte die Reduktion der Mobilität durchaus positive Folgen für Mensch und Umwelt. Die Lebensqualität könnte verbessert werden. Der motorisierte Verkehr stößt große Mengen von Schadstoffen aus, trägt zur Klimaänderung bei, und täglich kommen dabei weltweit so viele Menschen um, wie wenn fünf Großraumflugzeuge

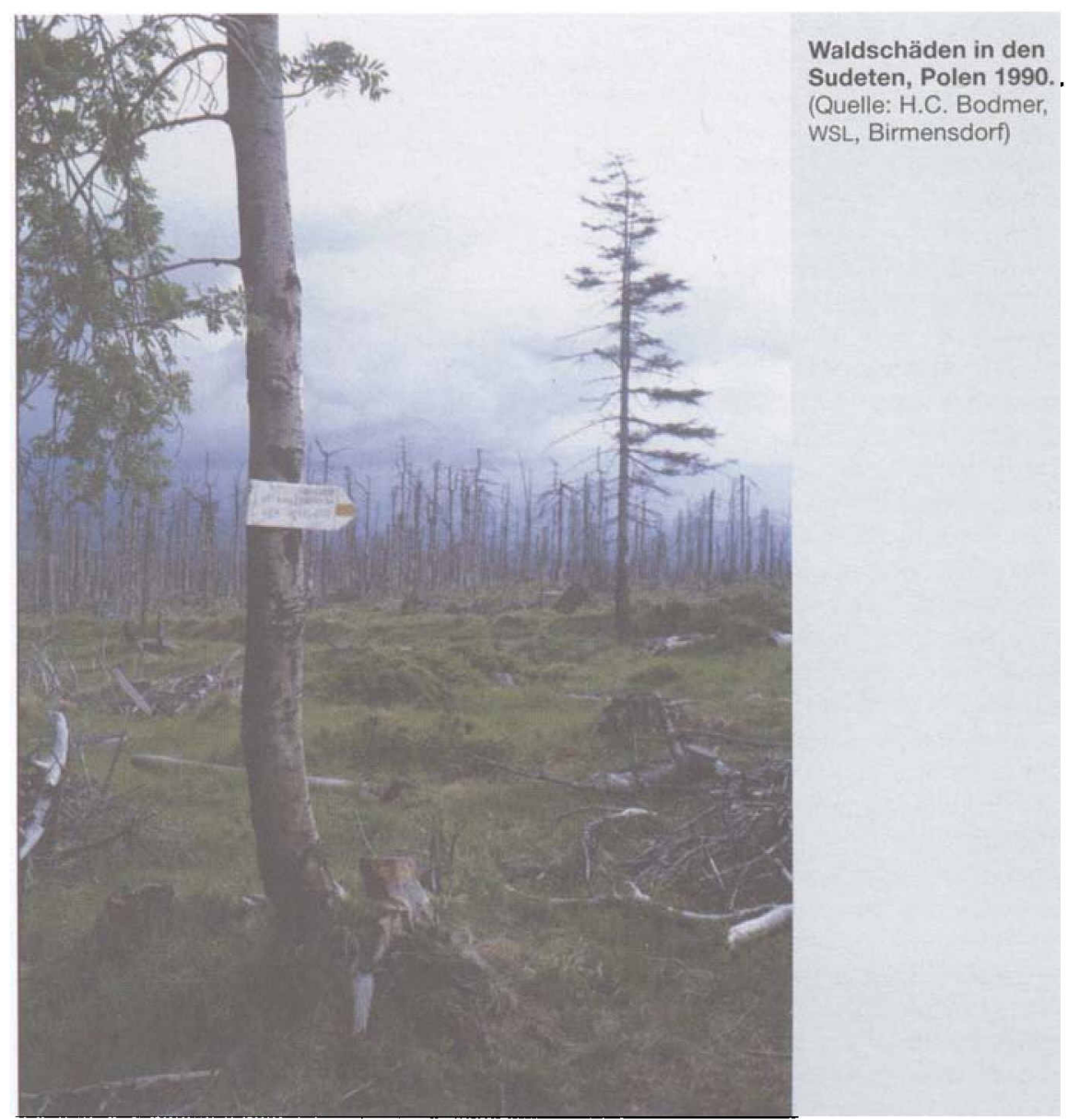

mit je 300 Personen abstürzen würden. Träfe letzteres ein, so flöge wohl niemand mehr. Wir sind offensichtlich Meister im Verdrängen.

Und was ist nun vom "Waldsterben" aus wissenschaftlicher Sicht geblieben? - Die damalige Extrapolation vom Riesen- und Erzgebirge mit sehr hohen Schwefeldioxid- und Säurebelastungen auf die Alpen war räumlich wie sachlich sehr heikel, und die Bäume waren resistenter als angenommen. Die Erwartung an die Forschung um schnelle Antworten war angesichts der Komplexität der Systeme zu hoch, kurzfristige Antworten waren nicht möglich. Die Waldökosystemforschung ist langfristig anzulegen. Man muß demgemäß im nachhinein von einer $\mathrm{zu}$ vereinfachten Waldsterbensdebatte reden. Auch mancher Wissenschaftler ließ sich in der herr- 
schenden Ungewißheit zu gewagten Aussagen hinreißen.

- In der Schweiz dürften die Luftschadstoffe kaum akute Schäden an Bäumen bewirken. Die beobachteten Kronenverlichtungen können natürliche Ursachen haben und lassen sich nicht kausal mit Schaden oder Krankheit verbinden. Demgemäß ist das "Schadstufen"-Konzept fragwürdig. In der Schweiz wurde die willkürlich wertende "Schadstufen"Terminologie mit dem Sanasilva-Bericht 1997 aufgegeben. Bereits ab 1992 hat die Eidgenössische Forschungsanstalt für Wald, Schnee und Landschaft (WSL) auf die unklaren Abgrenzungen zwischen 'gesund' und 'geschädigt' aufmerksam gemacht.

- Ausgelöst durch die "Waldsterbens"Forschung hat das Wissen um das Ökosystem Wald stark zugenommen, insbesondere was Stoffflüsse, Baumphysiologie und Wurzeln betrifft. Die Forschung ging vom Sichtbaren, nämlich von der Baumkrone, in das Unsichtbare, in die verborgenen Prozesse im Boden. Die kritischen Belastungsgrenzen für Säuren, Stickstoff und Ozon werden in der Schweiz weiträumig überschritten. Dies führt aber nicht zu akuten Schädigungen, sondern zu nachteiligen Langzeitveränderungen. Dies ist einer der wichtigsten Wissensfortschritte der Waldschadensforschung. Wir können also heute abschätzen, welche Wirkung Schadstoffeinträge langfristig im Ökosystem Wald haben, welche Wälder empfindlich und welche eher resistent sind. Das Verständnis rund um das Ökosystem Wald hat also stark zugenommen; es ist dies eine Umwegrentabilität der Waldschadensforschung.
- Es wurden umweltpolitisch richtige Maßnahmen getroffen, aber mit wissenschaftlich nicht haltbaren Begründungen, das heißt verkürzt und plakativ gesagt: Die Therapie war richtig, trotz falscher Diagnose. Es gibt also Erfolge der Luftreinhaltepolitik auszuweisen; so haben wir beispielsweise heute noch etwa ein Viertel der Schwefeldioxidbelastung von derjenigen vor zwanzig Jahren.

Das Szenario "Waldsterben" traf also nicht ein, doch noch immer belasten wir die Mitwelt stärker, als es $\mathrm{jhr}$ und uns langfristig zuträglich ist. Wir müssen daher lernen, mit Langzeitrisiken umzugehen.

[1] W. Zierhofer: Umweltforschung und Öffentlichkeit: Das Waldsterben und die kommunikativen Leistungen von Wissenschaft und Massenmedien, Westdeutscher Verlag, Opladen (1998).

\section{Zehn Jahre Erfolgspolitik zum Schutz der Ozonschicht - nicht aber der globalen Atmosphäre}

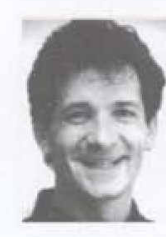

Thomas Peter

Professor für Atmosphärenchemie und Leiter der Gruppe Atmosphärenchemie am Institut für Atmosphäre und Klima, ETH Zürich

E-Mail: thomas.peter@ethz.ch www.iac.ethz.ch/en/groups/peter/
Abstract: In Copenhagen 1992, the international community finally decided to curb chlorine emissions of industrial gases in order to protect the ozone layer. This amendment to the 1987 "Montreal Protocol" could mark a paradigm shift in international environmental policy. However, our current climate policy ignores that future climate change may lead to a massive delay in ozone recovery. (or zehn Jahren, 1992, entschied sich die internationale Staatengemeinschaft in Kopenhagen unisono, dem anthropogen bedingten Anstieg der Chlorkonzentration in der globalen Atmosphäre endlich Einhalt zu gebieten. Chlor war bis dahin auf etwa das Fünffache des natürlichen Wertes angestiegen. Kopenhagen bedeutete eine wesentliche Verschärfung des erst fünf Jahre zuvor vereinbarten "Montrealer Protokolls" zum Schutz der Ozonschicht und könnte einen Paradigmenwechsel in der internationalen Umweltpolitik markieren. Doch während die beschlossenen Vereinbarungen rechtlich Bestand haben und rigoros verfolgt werden, ist die Politik schnellebig. In Zeiten flauer Konjunktur rücken ökologische Belange rasch in den Hintergrund, wie die gegenwärtige Krise bei den internationalen Klimaverhandlungen zeigt. Unser "business as usual" in der Klimafrage ignoriert, daß die Ozonschicht über den dichtbesiedelten nördlichen Teilen Europas, Asiens und Amerikas trotz des sinkenden atmosphärischen Chlorgehaltes in den kommenden Jahrzehnten durch anthropogene Klimaveränderungen weiter abnehmen könnte. Mit Lebensdauern von typischerweise hundert Jahren bei vielen ozonzerstörenden und klimaverändernden Gasen sind die zugrundeliegenden globalen Probleme extrem langlebig, und jedes Jahr des Aufschubs einer Selbstbegrenzung bei den Treibhausgas-Emissionen heute schlägt sich unvermeidbar in vielen Jahren Lösungsverzug zur Zeit unserer Enkelkinder nieder.

\section{Naturwissenschaftliche Forschung 1992-2002}

In weitgehender Unkenntnis der ablaufenden physikalischen und chemischen Prozesse in der antarktischen Stratosphäre kam die Entdeckung des Ozonlochs 1985 als ein völlig unerwartetes Ereignis nicht nur für die breite Öffentlichkeit, sondern auch für die Fachwelt [']. Nach intensiven wissenschaftlichen Auseinandersetzungen über die Ursachen des Ozonlochs setzte sich Ende der achtziger Jahre die anthropogen-chemische Erklärung durch. Aus Fluorchlorkohlenwasserstoffen (FCKW) stammendes Chlor konnte in Messungen über der Antarktis als Hauptverursacher der Ozonzerstörung nachgewiesen werden [2]. Während das Ozonloch im antarktischen Frühjahr aufgrund spezieller meteorologischer Bedingungen ein extrem klares Signal darstellt, ist die Situation in den dichtbesiedelten mittleren und hohen nördlichen Breiten weit weniger klar. Dort hätte ein Ozonloch vom Ausmaß des antarktischen Phänomens katastrophale Folgen.

Dementsprechend konzentrierte sich die stratosphärische Ozonforschung auf die Arktis und die nördlichen mittleren Breiten. Wesentliche Ergebnisse in der Zeit von 1992 bis 2002 betreffen das Verständnis folgender Entwicklungen: (1) Der Schwund der Ozonschicht in den nördlichen mittleren Breiten, also im Bereich $30^{\circ} \mathrm{N}$ bis $60^{\circ} \mathrm{N}$, hat an Signifikanz gewonnen; 